\title{
Codebraid: Live Code in Pandoc Markdown
}

\author{
Geoffrey M. Poore ${ }^{* *}$
}

\begin{abstract}
Codebraid executes code blocks and inline code in Pandoc Markdown documents as part of the document build process. Code can be executed with a built-in system or Jupyter kernels. Either way, a single document can involve multiple programming languages, as well as multiple independent sessions or processes per language. Because Codebraid only uses standard Pandoc Markdown syntax, Pandoc handles all Markdown parsing and format conversions. In the final output document produced by Pandoc, a code chunk can be replaced by a display of any combination of its original Markdown source, its code, the stdout or stderr resulting from execution, or rich output in the case of Jupyter kernels. There is also support for programmatically copying code or output to other parts of a document.
\end{abstract}

Index Terms—reproducibility, dynamic report generation, literate programming, Python, Pandoc, Project Jupyter

\section{Introduction}

Scientific and technical documents are increasingly written with software that allows a mixture of text and executable code, such as the Jupyter Notebook [KRKP $\left.{ }^{+} 16\right]$, knitr [YX15], and Orgmode Babel [SD11], [SDDD12]. Writing with such tools can enhance reproducibility, simplify code documentation, and aid in automating reports.

This paper introduces Codebraid, which allows executable code within Pandoc Markdown documents [JG19], [JM19]. Codebraid is developed at https://github.com/gpoore/codebraid and is available from the Python Package Index (PyPI). It allows Markdown code blocks like the one below to be executed during the document build process. In this case, the ".cb.run" tells Codebraid to run the code and include the output.

‥python . cb.run

print("Running code within *Markdown! *")

The final document contains the code's output, interpreted as if it had been entered directly in the original Markdown source:

Running code within Markdown!

A document using Codebraid can be converted from Markdown into any of the many formats supported by Pandoc, such as HTML, Microsoft Word, LaTeX, and PDF. Codebraid delegates all Markdown parsing and format conversions to Pandoc, so it does not introduce any special restrictions on what is possible with a Pandoc Markdown document. This close integration with Pandoc also means that Codebraid can be extended in the future to work with additional document formats beyond Markdown.

* Corresponding author: gpoore@uu.edu

+ Union University

Copyright $\odot 2019$ Geoffrey M. Poore. This is an open-access article distributed under the terms of the Creative Commons Attribution License, which permits unrestricted use, distribution, and reproduction in any medium, provided the original author and source are credited.
Codebraid includes a built-in code execution system. It can also use Jupyter kernels $\left[\mathrm{KRKP}^{+} 16\right]$ to execute code. The first code block that is executed with a given language can specify a kernel. In the example below, the ". cb. nb" tells Codebraid to run the code and provide a "notebook" display that shows both code and output, while "jupyter_kernel" specifies a kernel.

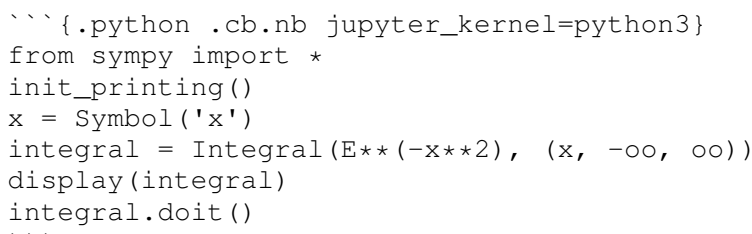

Because a Jupyter kernel was used to run the code, the result includes rich output in the form of rendered LaTeX math, just as it would in a Jupyter notebook:

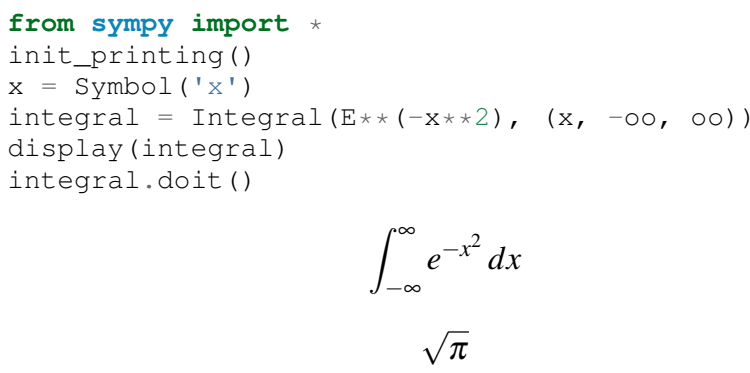

$$
\int_{-\infty}^{\infty} e^{-x^{2}} d x
$$$$
\sqrt{\pi}
$$

The next section provides an example of the document build process with Codebraid. This is followed by an overview of Codebraid features and capabilities. Finally, the Comparison considers Codebraid in the context of knitr, Pweave, Org-mode Babel, and the Jupyter Notebook.

\section{Building a simple Codebraid document}

A simple Pandoc Markdown document that runs code with Codebraid is shown below.

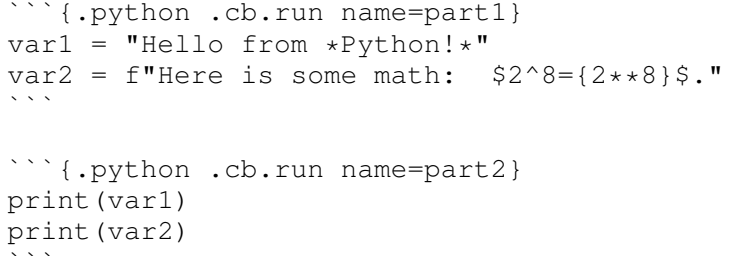

If this were a normal Pandoc document, converting it into a format such as reStructuredText could be accomplished by running

pandoc --from markdown --to rst file.md 
Using Codebraid to execute code as part of the document conversion process is simply a matter of replacing pandoc with codebraid pandoc:

codebraid pandoc --from markdown --to rst file.md

The codebraid executable is available from the Python Package Index (PyPI); development is at https://github.com/gpoore/ codebraid. By default, code is executed with Codebraid's built-in code execution system. This can easily be swapped for a Jupyter kernel, as shown in the Introduction and discussed in greater detail in Jupyter kernels.

When this codebraid pandoc command is executed, the original Markdown shown above is converted into Codebraidprocessed Markdown:

Hello from *Python!*

Here is some math: $\$ 2^{\wedge} 8=256 \$$.

This processed Markdown is then converted into the final reStructuredText, rendering as

Hello from Python! Here is some math: $2^{8}=256$.

By default, the output of code executed with cb.run is interpreted as Markdown. It is possible to show the output verbatim instead, as discussed later.

In this example, the code is simple enough that it could be executed every time the document is built, but that will often not be the case. By default, Codebraid caches all code output, and code is only re-executed when it is modified. This can be changed by building with the flag--no-cache.

\section{Pandoc code attribute syntax}

Pandoc Markdown defines an attribute syntax for inline code and code blocks. Codebraid uses this to designate which code blocks should be executed and provide options. Code attributes have the general form

\{\#id.class1.class2 key1=value1 key2=value2

If code with these attributes were converted into HTML, \#id becomes an HTML id for the code, anything with the form . class specifies classes, and space-separated key-value pairs provide additional attributes. Although key-value pairs can be quoted with double quotation marks, Pandoc allows most characters except the space and equals sign unquoted. Other output formats such as LaTeX use attributes in a largely equivalent manner.

Pandoc uses the first class to determine the language name for syntax highlighting, hence the . python in the example in the last section. Codebraid uses the second class to specify a command for processing the code. All Codebraid commands are under a $\mathrm{cb}$ namespace to prevent unintentional collisions with normal Pandoc attributes. In the example, cb.run indicates that code should be run, stdout should be included and interpreted as Markdown, and stderr should be displayed in the event of errors. If a Jupyter kernel were in use, rich output such as plots would also be included. Finally, the name keyword is used to assign a unique name to each piece of code. This allows the code to be referenced elsewhere in a document to insert any combination of its Markdown source, code, stdout, stderr, and rich output (for Jupyter kernels).

\section{Creating examples}

The example in Building a simple Codebraid document was actually itself an example of using Codebraid. This paper was written in Markdown, then converted to reStructuredText via Codebraid with Pandoc. Finally, the reStructuredText was converted through LaTeX to PDF via Docutils [DG16]. The two code blocks in the example were only entered in the original Markdown source of this paper a single time, and Codebraid only executed them a single time. However, with Codebraid's copy-paste capabilities, it was possible to display the code and output at other locations in the document programmatically.

The rendered output of the two code blocks is shown at the very end of the earlier section. This is where the code blocks were actually entered in the original Markdown source of this paper, and where they were executed.

Recall that both blocks were given names, part 1 and part 2. This enables any combination of their Markdown source, code, stdout, and stderr to be inserted elsewhere in the document. At the beginning of the earlier section, the Markdown source for the blocks was shown. This was accomplished via

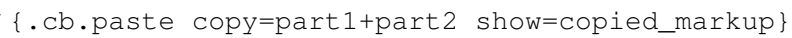

The $\mathrm{cb}$.paste command inserts copied data from one or more code chunks that are specified with the copy keyword. Meanwhile, the show keyword controls what is displayed. In this case, the Markdown source of the copied code chunks was shown. Since the $\mathrm{cb}$.paste command is copying content from elsewhere, it is used with an empty code block. Alternatively, a single empty line or a single line containing an underscore is allowed as a placeholder.

Toward the end of the earlier section, the verbatim output of the Codebraid-processed Markdown was displayed. This was inserted in a similar manner:

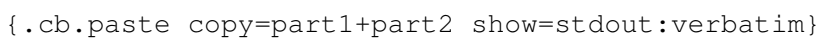

The default format of stdout is verbatim, but this was specified just to be explicit. The other option is raw (interpreted as Markdown).

Of course, all Markdown shown in the current section was itself inserted programmatically using $\mathrm{cb}$.paste to copy from the earlier section. However, to prevent infinite recursion, the next section is not devoted to explaining how this was accomplished.

\section{Other Codebraid commands}

The commands cb.run and cb.paste have already been introduced. There are three additional commands.

The cb.code command simply displays code, like normal inline code or a code block. It primarily exists so that normal code can be named, and then accessed later. cb. paste could be used to insert the code elsewhere, perhaps combined with code from other sources via something like $c o p y=c o d e 1+c o d e 2$. It would also be possible to run the code elsewhere:

\section{$\{$. cb. run copy $=$ code $1+$ code 2$\}$}

When copy is used with cb.run, or another command that executes code, only code is copied, and everything proceeds as if this code had been entered directly in the code block.

The cb.expr command only works with inline code, unlike other commands. It evaluates an expression and then prints a string representation, which is interpreted as Markdown. For example, 
$2 \star * 128^{`}\{$.python $\cdot$ cb.expr\}

produces

340282366920938463463374607431768211456

As this demonstrates, Pandoc code attributes for inline code immediately follow the closing backtick(s). While this sort of a "postfix" notation may not be ideal from some perspectives, it is the cost of maintaining full compatibility with Pandoc Markdown syntax.

Finally, the $\mathrm{cb}$. nb command runs code and provides a "notebook" display. For inline code, $\mathrm{cb}$. nb is like cb.expr except that it displays rich output or verbatim text. For code blocks, $\mathrm{cb}$. nb displays code followed by verbatim stdout. If there are errors, stderr is also included automatically. When Codebraid is used with a Jupyter kernel, rich outputs such as plots are included as well. This was demonstrated in the Introduction.

\section{Display options}

There are two code chunk keywords that govern display, show and hide. These can be used to override the default display settings for each command.

show takes any combination of the following options: markup (display Markdown source), code (display code being executed), stdout, stderr, and none. There is also rich_output when a Jupyter kernel is used to execute code. Multiple options can be combined, such as show $=$ code+stdout + st derr. Code chunks using copy can employ copied_markup to display the Markdown source of the copied code chunk. When the cb.expr command is used, the expression output is available via expr. Using show completely overwrites the existing display settings.

The display format can also be specified with show. For stdout, stderr, and expr, there are three formats: raw (interpreted as Markdown), verbatim, or verbatim_or_empty (verbatim if there is output, otherwise a space or empty line). For example, show=stdout: $r a w+$ stderr: verbatim. While a format can be specified for markup and code, only the default verbatim is permitted. For rich_output, the output representation (MIME type) can be selected. Thus, show=rich_output: png selects a PNG image representation.

hide takes the same options as show, except that none is replaced by $\mathrm{a} l \mathrm{l}$ and formats are not specified. Instead of overriding existing settings like show, hide removes the specified display options from those that currently exist.

\section{Codebraid code execution system}

Codebraid currently provides two options for executing code: a built-in code execution system which is used by default and Jupyter kernels. Jupyter kernels are demonstrated in the next section. This section describes the built-in system, which currently supports Python 3.5+, Julia, Rust, R, Bash, and JavaScript. Any combination of these languages can be used within a single document. While the built-in system currently lacks Jupyter kernel features like rich output, it is nearly identical to extracting the code from a document, concatenating it, and executing it via the standard interpreter or compiler. As a result, it has low overhead and produces the same output as would have been generated by a separate source file.

\section{Overview}

The code from each code chunk is inserted into a template before execution. The template writes delimiters to stdout and stderr at the beginning of each code chunk. These delimiters are based on a hash of the code to avoid the potential for collisions. Once execution is complete, Codebraid parses stdout and stderr and uses these delimiters to associate output with individual code chunks. This system is a more advanced variant of the one I created previously in PythonTeX [GMP15].

By default, code must be divided into complete units. For example, a code block must contain an entire loop, or an entire function definition. (This restriction can be relaxed with the codechunk keyword complete; see Incomplete units of code later.) If a code chunk is not complete (and this is not indicated), then the incomplete code will interfere with writing the delimiters.

To address this, each individual delimiter is unique, and is tracked individually by Codebraid. If incomplete code interferes with the template to produce an error, Codebraid can use any existing stderr delimiters plus parsing of stderr to find the source and generate an appropriate error message. If the code does not produce an error, but prevents a delimiter from being written or causes a delimiter to be written multiple times or not at the beginning of a line, this will also be detected and traced back. Under normal conditions, interfering with the delimiters without detection requires conscious effort.

\section{Adding languages}

Adding support for additional languages is simply a matter of creating the necessary templates and putting them in a configuration file. Basic language support can require very little, essentially just code for writing the delimiters to stdout and stderr. For example, Bash support is based on this three-line template:

printf " $\backslash n\{$ stdout_delim\} $\backslash n "$

printf " $\backslash n\{$ stderr_delim $\} \backslash n ">\& 2$

\{code

The Bash configuration file also specifies that the file extension . sh should be used, and provides another four lines of template code to enable $\mathrm{cb}$.expr. So far, the longest configuration file, for Rust, is less than fifty lines-counting empty lines.

\section{Stderr}

Because code is typically inserted into a template for execution, if there are errors the line numbers will not correspond to those of the code that was extracted from the document, but rather to those of the code that was actually executed. Codebraid tracks line numbers during template assembly, so that executed line numbers can be converted into original line numbers. Then it parses stderr and corrects line numbers. An example of an error produced with $\mathrm{Cb}$. nb with Python is shown below. Notice that the line number displayed is correct.

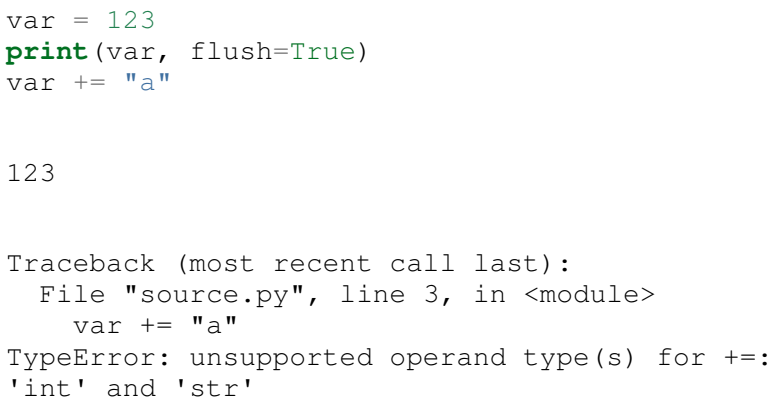


Since line numbers in errors and warnings correspond to those in the code entered by the user, and since anything written to stderr is displayed by default next to the code that caused it, debugging is significantly simplified. In many cases, this even applies to compile errors for a language like Rust.

\section{Jupyter kernels}

Using a Jupyter kernel instead of the built-in code execution system is as simple as adding jupyter_kernel $=<$ name $>$ to the first code chunk for a language (or, as discussed later, to the first code chunk of a named session):

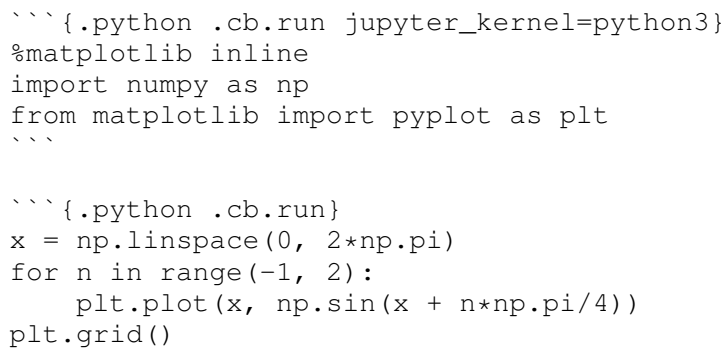

Notice that jupyter_kernel was only needed (and only allowed) for the first code chunk. The second code chunk is still using the same language (. python), so it shares the same kernel. This Markdown results in a plot, just as it would within a Jupyter notebook. Because $\mathrm{cb}$.run was used rather than $\mathrm{cb} . \mathrm{nb}$, code is not displayed and only the plot is shown:

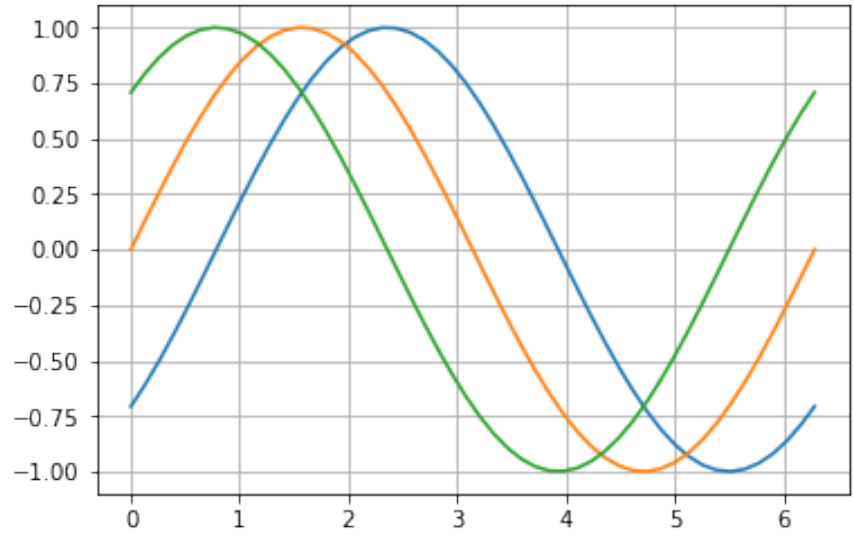

The built-in code execution system allows multiple languages within a single document. This is also possible when Jupyter kernels are used instead. A single document can involve multiple kernels. Multiple independent sessions for the same kernel type are also possible when jupyter_kernel is combined with session (described in the next section). Of course, kernel features like IPython magics [IDT19a] are fully functional as well.

\section{Advanced code execution}

Ideally, executable code should be arranged within a document based on what is best for the reader, rather than in a manner dictated by limitations of the tooling. Several options are provided to maximize the flexibility of code presentation.

\section{Incomplete units of code}

By default, Codebraid requires that code be divided into complete units. For example, a code block must contain an entire loop, or an entire function definition. Codebraid's built-in code execution system can detect the presence of an incomplete unit of code because it interferes with stdout and stderr processing, in which case Codebraid will raise an error. Attempting to run an incomplete unit of code with a Jupyter kernel will also result in an error.

The complete keyword allows incomplete units of code. While this increases the flexibility of code layout, it also means that any output will not be shown until the next complete code chunk.

The Markdown for a somewhat contrived example that demonstrates these capabilities is shown below, along with its output. While this example uses Codebraid's code execution system, exactly the same result is obtained by using a Jupyter kernel.

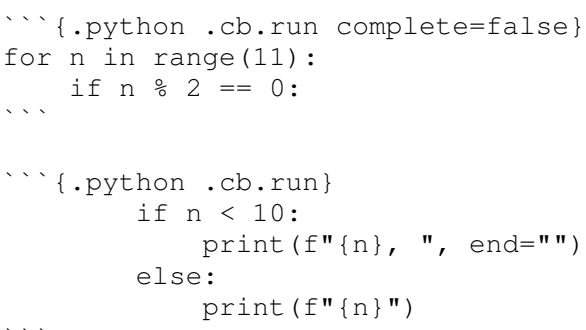

$0,2,4,6,8,10$

\section{Sessions}

By default, all code for a language is executed within a single default session, so variables and data are shared between code chunks. It can be convenient to separate code into multiple sessions when several independent tasks are being performed, or when a long calculation is required but the output can easily be saved and loaded by separate code for visualization or other processing. The session keyword makes this possible. Session names are restricted to valid Python identifiers. For example,

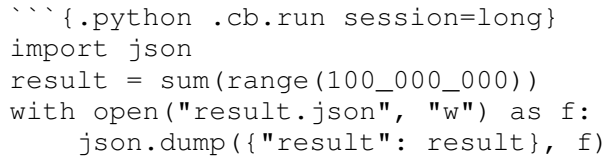

Sessions work with both Codebraid's built-in code execution system and Jupyter kernels. For example, it is possible to have multiple independent sessions with a python3 kernel within a single document.

All sessions are currently executed in serial. In the future, support for parallel execution may be added.

\section{Outside main ()}

Codebraid's built-in code execution system runs code by inserting it into a template. The template allows stdout and stderr to be broken into pieces and correctly associated with the code chunks that created them. For a language like Python under typical usage, complete eliminates the few limitations of this approach. However, the situation for a compiled language with a main () function is more complex.

Codebraid includes support for Rust. By default, code is inserted into a template that defines a main() function. Thus, a code block like

\footnotetext{
$\cdots\{$.rust . cb.run

let $\mathrm{x}=$ "Greetings from *Rust $! *$;
} 
println! (" \{\}", $\mathrm{x})$

can run to produce

\section{Greetings from Rust!}

In some situations, it would be convenient to completely control the definition of the main() function and add code outside of main(). The outside_main keyword makes this possible. All code chunks with outside_main=true at the beginning of a session are used to overwrite the beginning of the main() template (everything before main()), while any chunks with outside_main=true at the end of the session are used to overwrite the end of the main () template (everything after main ( ) ). If all code chunks have outside_main=true, then all of Codebraid's templates are completely omitted, and all output is associated with the final code chunk. The example below demonstrates this option.

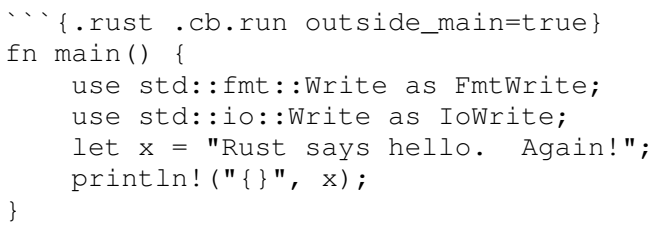

Rust says hello. Again!

\section{Working with external files}

Though Codebraid is focused on embedding executable code within a document, there will be times when it is useful to interact with external source files. Since Codebraid's built-in code execution system processes code with a programming language's standard interpreter or compiler, normal module systems are fully compatible; for example, in Python, import works normally. Of course, this is also true when working with Jupyter kernels. Codebraid provides additional ways to work with external files via the include_file option.

When include_file is used with the cb. code command, an external source file is simply included and displayed. It is possible to include only certain line ranges using the additional option include_lines, or only part of a file that matches a regular expression via include_regex. For example,

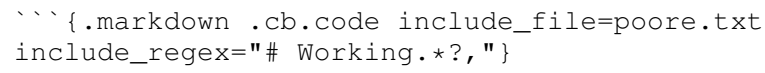

includes the original Markdown source for this paper, and then uses a regular expression to display only the first few lines of this current section on working with external files:

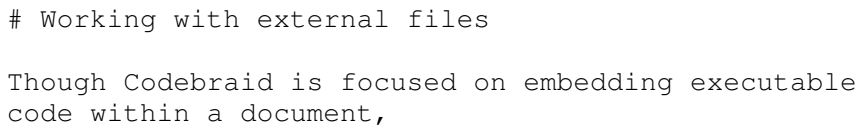

Since the cb.code command is including content from elsewhere, it is used with an empty code block. Alternatively, a single empty line or a single line containing an underscore is allowed as a placeholder.

This example included part of a file using a single regular expression. There are also options for including everything starting with or starting after a literal string or regular expression, and for including everything before or through a literal string or regular expression.

The include_file option works with commands that execute code as well. For instance,

‥python . cb.run include_file=code.py

would read in the contents of an external file "code.py" and then run it in the default Python session, just as if it had been entered directly within the Markdown file.

\section{Comparison}

To put Codebraid in context, this section provides a comparison with knitr, Pweave, Org-mode Babel, and the Jupyter Notebook. The comparison focuses on the default features of each program. Extensions for these programs and additional programs with similar features are summarized in the Appendix.

knitr

knitr [YX15] provides powerful $\mathrm{R}$ evaluation in Markdown, LaTeX, HTML, and other formats. It was inspired by Sweave [FL02], which allows $\mathrm{R}$ in LaTeX. The reticulate $\left[\mathrm{AUT}^{+} 19\right]$ and JuliaCall [CL19] packages for $\mathrm{R}$ have given knitr significant Python and Julia capabilities as well, including the ability to convert objects between languages. knitr is commonly used with the RStudio IDE, which provides a two-panel source-and-output preview interface as well as a notebook-style mode with inline display of results.

While knitr provides superior support for R, Codebraid focuses on providing more capabilities for other languages. knitr runs all R, Python, and Julia code in language-specific sessions, so data and variables are shared between code chunks. For all other languages, each code chunk is run in a separate process and there is no such continuity. Codebraid's built-in code execution system is designed to allow any language to share a session between multiple code chunks, and Jupyter kernels provide equivalent capabilities. R, Python, and Julia are limited to a single shared session each with knitr. Codebraid allows multiple sessions for all supported languages. This allows independent computations to be divided into separate sessions and only re-executed when necessary.

Once code is executed, Codebraid and knitr provide similar basic features for displaying the code and its output. knitr has more advanced options for formatting output, such as customizing plot appearance, converting plots into figures with captions, or combining plots into an animation.

The two programs take different approaches to extracting code from Markdown documents. knitr uses the custom R Markdown [RSt18] syntax to designate code that should be executed. It extracts inline code and code blocks from the original Markdown source using a preprocessor, then inserts the code's output into a copy of the document that can subsequently be processed with Pandoc. Because the preprocessor is based on simple regex matching, it does not understand Markdown comments and will run code in a commented-out part of a document. Writing tutorials that show literal knitr code chunks can involve inserting empty strings, zero-width spaces, line breaks, or Unicode escapes to avoid the preprocessor's tendency to execute code [YX19], [Hov17]. With Codebraid, Pandoc is used to convert a Markdown document into Pandoc's abstract syntax tree (AST) representation. Code extraction and output insertion are performed as operations on the 
AST, and then Pandoc converts the modified AST into the final output document. This has the advantage that Pandoc handles all parsing and conversion, at the cost of running Pandoc multiple times.

\section{Pweave}

Pweave [MP16] is inspired by Sweave [FL02] and knitr [YX15], with a focus on Python in Markdown and other formats like LaTeX and reStructuredText. Pweave uses a custom Markdown syntax similar to knitr's for designating code blocks that should be executed, with many similar features and options. It also extracts code from Markdown documents with a simple preprocessor. Code is executed with a single Jupyter kernel. Any kernel can be used; the default is python3. Rich output like plots can be included automatically.

Like knitr, Pweave provides some more advanced options for display formatting that Codebraid lacks, primarily related to figures. Codebraid has advantages in three areas. Code execution is more flexible since it allows multiple Jupyter kernels per document and multiple independent sessions per kernel, in addition to the built-in code execution system. Since Codebraid uses Pandoc for all Markdown parsing, it avoids the limitations of a preprocessor. Codebraid also provides a broader set of display capabilities, including the ability to programmatically copy and display code or its output into other parts of a document.

\section{Org-mode Babel}

Babel [SD11], [SDDD12] allows code blocks and inline code in Emacs Org-mode documents to be executed. Any number of languages can be used within a single document. By default, each code chunk is executed individually in its own process. For many interpreted languages, it is also possible to run code in a session so that data and variables persist between code chunks. In those cases, multiple sessions per language are possible. Any combination of code and its stdout can be displayed. Stdout can be shown verbatim or interpreted as Org-mode, HTML, or LaTeX markup. For some languages, such as gnuplot, graphical output can also be captured and included automatically.

Babel can function as a meta-programming language for Org mode. A code chunk can be named, and then a later code chunk-potentially in a different language — can access its output by name and perform further processing. Similarly, there are literate programming capabilities that allow a code chunk to copy the source of one or more named chunks into itself, essentially serving as a template, before execution.

Codebraid is like a Markdown-based Babel with additional code execution capabilities but without some of the metaprogramming and literate programming options. Codebraid allows sessions for all languages, not just for some interpreted languages. It provides broad support for rich output like plots through Jupyter kernels. Stderr can also be displayed. While Codebraid currently lacks a system for passing output between code chunks, it does provide some literate-programming style capabilities for code reuse.

\section{Jupyter Notebook}

The Jupyter (formerly IPython) Notebook $\left[\mathrm{KRKP}^{+} 16\right]$ provides a browser-based user interface in which a document is represented as a series of cells. A cell may contain Markdown (which is converted into HTML and displayed when not being edited), raw text, or code. Code is executed by language-specific backends, or kernels. Well over one hundred kernels are available beyond Python, including Julia, R, Bash, and even compiled languages like C++ and Rust [Jup19c]. Jupyter kernels are often used with the Jupyter Notebook, but they can also function as a standalone code execution system.

A Jupyter Notebook can only have a single kernel, and thus only a single primary programming language with a single session or process. This means that dividing independent computations into separate sessions or processes is typically not as straightforward as it might be in Org-mode Babel or Codebraid. However, the interactive nature of the notebook often reduces the impact of this limitation, and can actually be a significant advantage. Code cells can be run one at a time; a single code cell can be modified and run again without re-executing any previous code cells.

Some kernels include support for interacting with additional languages. The IPython kernel [IDT19b] has $\%$ script and similar "magics" [IDT19a] that allow single cells to be executed in a subprocess by another language. PyJulia [JIdt19] and rpy2 [LGrc16] provide more advanced magics that allow an IPython kernel to interact with a single Julia or R session over a series of cells (see [MB18b] for examples).

While Codebraid lacks the Jupyter Notebook's interactivity, it does have several capabilities not present in the default Notebook. A Codebraid document can involve multiple Jupyter kernels, as well as multiple independent sessions per kernel. It can execute both code blocks and inline code; the Jupyter Notebook is limited to executing code in code cells. Code layout is more flexible with Codebraid because a code chunk can contain an incomplete unit of code, such as part of a loop or part of a function definition. This is possible even when working with Jupyter kernels. Codebraid also provides more flexible display options. It is possible to show any combination of code, stdout, stderr, or rich output in any order, and to select which form of rich output (MIME type) is shown. Code or its output can be copied programmatically, so code can be executed at one location in a document and its output displayed elsewhere.

\section{Conclusion}

Codebraid provides a unique and powerful combination of features for executing code embedded in Pandoc Markdown documents.

- Both code blocks and inline code can be executed.

- Code blocks are not required to contain complete units of code, like a complete loop or function definition.

- A single document can use multiple languages and multiple independent sessions per language. Any language can share a session between multiple code chunks. Independent computations can be divided into separate sessions and only re-executed when necessary.

- Code can be executed with the built-in system, or with Jupyter kernels which provide rich output such as plots.

- A code chunk can display any combination of its Markdown source, code, stdout, stderr, and rich output.

- It is easy to reuse code and its output programmatically with the paste functionality. It is also possible to include all or part of an external source file for display or execution.

- Because only standard Pandoc Markdown syntax is used, all Markdown parsing and document conversion can be delegated to Pandoc, and there are no issues with preprocessors that do not fully support Markdown syntax. 
There are several logical avenues for further development. One of the original motivations for creating Codebraid was to build on my previous work with PythonTeX [GMP15] to create a program that could be used with multiple markup languages. While Codebraid has focused thus far on Pandoc Markdown, little of it is actually Markdown-specific. It should be possible to work with other markup languages supported by Pandoc, such as LaTeX; all that is required is that Pandoc parses key-value attributes for some variant of a code block. Pandoc has recently added Jupyter notebooks to its extensive list of supported formats. Perhaps at some point it will be possible to convert a Codebraid document into a Jupyter notebook, perform some exploratory programming for a single session of a single language, and then convert back to Markdown.

Codebraid's caching system could also be improved in the future. Currently, caching is based only on the code that is executed. Adding a way to specify external dependencies such as data files would be beneficial.

\section{APPENDIX}

The Comparison focuses on the default features of knitr, Pweave, Org-mode Babel, and the Jupyter Notebook. This appendix summarizes extensions for these programs and additional programs with similar features.

\section{knitr extensions}

Though knitr does not include any support for Jupyter kernels, the knitron [FH16] and ipython_from_R [MW18b] packages have demonstrated that this is technically feasible.

\section{Software similar to Pweave}

The Comparison includes Pweave [MP16] because it is one of the most capable knitr-like systems for other languages. There are several other similar programs.

Weave.j1 [MP17], by the creator of Pweave, provides similar features for executing Julia code. It uses Julia to manage code execution rather than a Jupyter kernel.

knitpy [Kat18] describes itself as a port of knitr to Python. It uses knitr-style Markdown syntax, and provides code-block options to control basic code and output display. Other knitr-style options are not supported. Code is executed in a single Jupyter IPython kernel. stitch [TA16] is similar, drawing inspiration from knitr and knitpy. Compared to knitpy, it lacks options for customizing output display but has options for customizing figure display.

Knitj [JH19] is another Jupyter kernel-Markdown integration. Options for controlling display are contained in special comments in the first line of code within a code block, rather than in the code block's Markdown attributes. It focuses on producing HTML and includes efficient live preview capabilities.

There are also some comparable tools for reStructuredText. nb2plots can convert an ipynb notebook file into reStructuredText for Sphinx [MB18a]. When Sphinx builds the document, the code is still executed and plots are automatically included, so the live code and rich output of the notebook are not lost. It is possible to customize display by hiding code. The reStructuredText can also be converted to a Python source file or ipynb when that is desired.
The Jupyter Sphinx Extension [Jup19b] provides a jupyter-execute directive for running code in a Jupyter kernel. By default, code is executed within a single kernel, providing continuity. It is also possible to switch to a different kernel or switch to a different session using the same kernel type. Code and output (including rich output like plots) are displayed by default, but there are options for hiding code or output, or reversing their order. All code for a given Jupyter session can be converted into a script or a Jupyter notebook.

\section{Org-mode Babel extensions}

Packages like ob-ipython [GS17] and emacs-jupyter [NN19] allow Jupyter kernels $\left[\mathrm{KRKP}^{+} 16\right]$ instead of Babel's built-in code execution system. These add the capability to display error messages or rich output like graphics. The Emacs IPython Notebook [JMM19] takes a different approach by providing a complete Jupyter Notebook client in Emacs.

\section{Jupyter Notebook extensions and related software}

Some more general approaches to working around the limitation of one kernel per notebook are provided by the BeakerX polyglot magics [TSOS18], which support bidirectional autotranslation of data between languages, and the Script of Scripts (SoS) kernel [BP19], which acts as a managing kernel over multiple normal kernels.

It is possible to execute inline code within Markdown cells with the Python Markdown extension [Jup18c]. This treats Markdown cells as $\{$ expression\} $\}$-style templates so long as inline code is outside LaTeX equations. The extension also supports notebook export to other document formats with nbconvert [Jup19a] via a bundled preprocessor.

The Comparison does not consider hiding code or output in documents derived from Jupyter notebooks because this is possible with nbconvert [Jup19a] as well as extensions and other programs. Hiding code or output in exported documents is possible on a notebook-wide basis by configuring nbconvert with the TemplateExporter exclude options. It is also possible at the individual cell level by adding a tag to a cell (View, Cell Toolbar, Tags, then "Add tag") and then configuring nbconvert to use the desired TagRemovePreprocessor with a given tag. An alternative is to use extensions with their provided preprocessors or templates [Jup18a], [Jup18b], or employ a more comprehensive tool like Jupyter Book [LH19] that defines a set of tags for display customization.

The Comparison does not cover the Jupyter Notebook's JSONbased ipynb file format because there are multiple ways to work around its limitations. There are special diffing tools for ipynb files such as nbdime [MSA15]. It is also possible to save notebooks as Markdown files instead, or convert them to source code with Markdown in comments:

- Jupytext [MW18a], [MWtJT19] can convert Jupyter notebooks into Markdown or R Markdown (knitr), or into scripts in which code cells are converted into code while Markdown cells are converted into intervening comments. These formats can also be converted into Jupyter notebooks.

- notedown [AO16] can convert between Markdown and ipynb, and can also work with R Markdown documents.

- Pandoc [JM19] can convert to or from ipynb files. Notebooks, including cells along with their attributes, can be 
represented as standard Pandoc Markdown. podoc [CR18] is an earlier program for converting between ipynb and Pandoc's AST. It builds on the prior ipymd [CR16].

- The Hydrogen package [Hyd19] for the Atom text editor provides conversion between ipynb and source code plus comments. When such a source file is edited, Hydrogen can connect to a Jupyter kernel to display rich output inline within the editor. Similar capabilities are provided by the Python extension for VS Code [Mic19].

Of the programs listed above, Jupytext, notedown, and podoc provide ContentsManager subclasses for the Jupyter Notebook that allow it to seamlessly use Markdown as a storage format.

\section{RefERENCES}

[AO16] Aaron O'Leary. Convert IPython Notebooks to markdown (and back), 2016. URL: https://github.com/aaren/notedown.

[AUT ${ }^{+}$19] JJ Allaire, Kevin Ushey, Yuan Tang, Dirk Eddelbuettel, Bryan Lewis, and Marcus Geelnard. reticulate: R Interface to Python, 2019. URL: https://rstudio.github.io/reticulate/index.html.

[BP19] Bo Peng. SoS: Notebook environment for both interactive data analysis and batch data processing, 2019. URL: https://vatlab. github.io/sos-docs/.

[CL19] Changcheng Li. JuliaCall: an R package for seamless integration between R and Julia. The Journal of Open Source Software, 4(35):1284, 2019. doi:10.21105/joss.01284.

[CR16] Cyrille Rossant. Replace .ipynb with .md in the IPython Notebook, 2016. URL: https://github.com/rossant/ipymd.

[CR18] Cyrille Rossant. podoc, 2018. URL: https://github.com/podoc/ podoc.

[DG16] David Goodger. Docutils Project Documentation Overview, 2016. URL: http://docutils.sourceforge.net/docs/index.html.

[FH16] Fabian Hirschmann. knitron: knitr + IPython + matplotlib, 2016. URL: https://github.com/fhirschmann/knitron/.

[FL02] Friedrich Leisch. Sweave: Dynamic generation of statistical reports using literate data analysis. In Wolfgang Härdle and Bernd Rönz, editors, Compstat 2002 - Proceedings in Computational Statistics, pages 575-580. Physica Verlag, Heidelberg, 2002. ISBN 3-7908-1517-9. URL: http://www.stat.unimuenchen.de/ leisch/Sweave.

[GMP15] Geoffrey M. Poore. PythonTeX: reproducible documents with LaTeX, Python, and more. Computational Science \& Discovery, 8(1):014010, July 2015. URL: https://doi.org/10.1088\% 2F1749-4699\%2F8\%2F1\%2F014010, doi:10.1088/1749$4699 / 8 / 1 / 014010$.

[GS17] Greg Sexton. Readme, 2017. URL: https://github.com/ gregsexton/ob-ipython.

[Hov17] T. Hovorka. How to Show R Inline Code Blocks in R Markdown, 2017. URL: https://rviews.rstudio.com/2017/12/04/howto-show-r-inline-code-blocks-in-r-markdown/.

[Hyd19] Hydrogen Contributors. Hydrogen, 2019. URL: https://nteract. gitbooks.io/hydrogen/.

[IDT19a] The IPython Development Team. Built-in magic commands, 2019. URL: https://ipython.readthedocs.io/en/stable/interactive/ magics.html.

[IDT19b] The IPython Development Team. IPython Documentation, 2019. URL: https://ipython.readthedocs.io/en/stable/index.html.

[JG19] John Gruber. Markdown, 2002-2019. URL: https:// daringfireball.net/projects/markdown/.

[JH19] Jan Hermann. Knitj, 2019. URL: https://github.com/jhrmnn/ knitj.

[JIdt19] The Julia and IPython development teams. Welcome to PyJulia's documentation!, 2019. URL: https://pyjulia.readthedocs.io.

[JM19] John MacFarlane. Pandoc: a universal document converter, 2006-2019. URL: https://pandoc.org/.

[JMM19] John M. Miller. The Emacs IPython Notebook, 2019. URL: http://millejoh.github.io/emacs-ipython-notebook/.

[Jup18a] Jupyter Contrib Team. Codefolding, 2015-2018. URL: https://jupyter-contrib-nbextensions.readthedocs.io/en/latest/ nbextensions/codefolding/readme.html.
[Jup18b] Jupyter Contrib Team. Hide Input, 2015-2018. URL: https://jupyter-contrib-nbextensions.readthedocs.io/en/latest/ nbextensions/hide_input/readme.html.

[Jup18c] Jupyter Contrib Team. Unofficial Jupyter Notebook Extensions: Python Markdown, 2015-2018. URL: https://jupyter-contrib-nbextensions.readthedocs.io/en/latest/ nbextensions/python-markdown/readme.html.

[Jup19a] Jupyter Development Team. nbconvert: Convert Notebooks to other formats, 2015-2019. URL: https://nbconvert.readthedocs. io.

[Jup19b] Jupyter Development Team. Jupyter Sphinx Extension, 2019. URL: https://jupyter-sphinx.readthedocs.io.

[Jup19c] Jupyter Team. Jupyter kernels, 2019. URL: https://github.com/ jupyter/jupyter/wiki/Jupyter-kernels.

[Kat18] Jan Katins. knitpy: Elegant, flexible and fast dynamic report generation with python, 2018. URL: https://github.com/jankatins/ knitpy.

[KRKP $\left.{ }^{+} 16\right]$ Thomas Kluyver, Benjamin Ragan-Kelley, Fernando Pérez, Brian Granger, Matthias Bussonnier, Jonathan Frederic, Kyle Kelley, Jessica Hamrick, Jason Grout, Sylvain Corlay, Paul Ivanov, Damián Avila, Safia Abdalla, and Carol Willing. Jupyter Notebooks - a publishing format for reproducible computational workflows. In F. Loizides and B. Schmidt, editors, Positioning and Power in Academic Publishing: Players, Agents and Agendas, pages 87-90. IOS Press, 2016. doi:10.3233/978-161499-649-1-87.

[LGrc16] Laurent Gautier \& rpy2 contributors. Documentation for rpy2, 2008-2016. URL: https://rpy2.readthedocs.io.

[LH19] Sam Lau and Chris Holdgraf. Jupyter Book: Books with Jupyter and Jekyll, 2019. URL: https://jupyter.org/jupyter-book/intro.

[MB18a] Matthew Brett. nb2plots - the documentation that is not missing, 2016-2018. URL: http://matthew-brett.github.io/nb2plots/.

[MB18b] Matthias Bussonnier. I Python, You R, We Julia, 2018. URL: https://blog.jupyter.org/i-python-you-r-we-julia-baf064ca1fb6.

[Mic19] Microsoft. Working with Jupyter Notebooks in Visual Studio Code, 2019. URL: https://code.visualstudio.com/docs/python/ jupyter-support.

[MP16] Matti Pastell. Pweave - Scientific Reports Using Python, 20102016. URL: http://mpastell.com/pweave/.

[MP17] Matti Pastell. Weave.jl: Scientific Reports Using Julia. Journal of Open Source Software, 2(11), 2017. URL: http://joss.theoj.org/ papers/10.21105/joss.00204, doi:10.21105/joss.00204.

[MSA15] Martin Sandve Alnæs. nbdime - diffing and merging of Jupyter Notebooks, 2015. URL: https://nbdime.readthedocs.io/en/latest/ index.html.

[MW18a] Marc Wouts. Introducing Jupytext, 2018. URL: https:// towardsdatascience.com/introducing-jupytext-9234fdff6c57.

[MW18b] Marc Wouts. ipython_from_R: Communicate with jupyter kernels from R, 2018. URL: https://github.com/mwouts/ipython_ from_R.

[MWtJT19] Marc Wouts and the Jupytext Team. Jupyter notebooks as Markdown documents, Julia, Python or R scripts, 2018-2019. URL: https://jupytext.readthedocs.io/.

[NN19] Nathaniel Nicandro. An interface to communicate with Jupyter kernels in Emacs, 2019. URL: https://github.com/dzop/emacsjupyter.

[RSt18] RStudio Inc. R Markdown, 2016-2018. URL: https:// rmarkdown.rstudio.com/.

[SD11] E. Schulte and D. Davison. Active Documents with Org-Mode. Computing in Science Engineering, 13(3):66-73, May 2011. doi:10.1109/MCSE.2011.41.

[SDDD12] Eric Schulte, Dan Davison, Thomas Dye, and Carsten Dominik. A Multi-Language Computing Environment for Literate Programming and Reproducible Research. Journal of Statistical Software, 46(3):1-24, 1 2012. URL: http://www.jstatsoft.org/ v46/i03.

[TA16] Tom Augspurger. stitch, 2016. URL: https://pystitch.github.io/.

[TSOS18] LLC Two Sigma Open Source. BeakerX, 2018. URL: http: //beakerx.com/.

[YX15] Yihui Xie. Dynamic Documents with R and knitr. Chapman \& Hall/CRC Press, 2015.

[YX19] Yihui Xie. Frequently asked questions, 2005-2019. URL: https: //yihui.name/knitr/faq/. 